Korean J. Math. 21 (2013), No. 4, pp. 375-382

http://dx.doi.org/10.11568/kjm.2013.21.4.375

\title{
REGULAR CONVERGENCE SPACES
}

\author{
JIN WON PARK
}

\begin{abstract}
In this paper, I introduce the notion of regular convergence space and give some properties of this space. And I give some conditions for the regularity of continuous convergence structure.
\end{abstract}

\section{Introduction}

Many researchers have been tried to obtain convenient categories in which the exponential law exists. In this point of view, convergence spaces are considered as good spaces with the exponential laws. Since the concept of convergence spaces was introduced, many studies about this space have been performed. In this paper, I introduce the notion of regular convergence space and investigate some properties of this space. And, for convergence spaces $X$ and $Y$, I give some conditions for the regularity of continuous convergence structure on function spaces $C(X, Y)$ using the cartesian closedness.

\section{Preliminaries}

In this section, we collect some basic definitions and known results on convergence spaces [1].

Received July 18, 2013. Revised September 13, 2013. Accepted September 23, 2013.

2010 Mathematics Subject Classification: 54A20.

Key words and phrases: regular convergence space, continuous convergence.

This work was supported by Jeju National University (2013).

(c) The Kangwon-Kyungki Mathematical Society, 2013.

This is an Open Access article distributed under the terms of the Creative commons Attribution Non-Commercial License (http://creativecommons.org/licenses/by -nc/3.0/) which permits unrestricted non-commercial use, distribution and reproduction in any medium, provided the original work is properly cited. 
Jin Won Park

For any set $X$, we denote by $F(X)$ the set of all filters on $X$, and by $P(F(X))$ the power set of $F(X)$.

Definition 2.1. Let $X$ be a set. A map $c: X \rightarrow P(F(X))$ is said to be a convergence structure if the following properties hold for any point $x \in X:$

(1) $\dot{x} \in c(x)$;

(2) if $\mathcal{F} \in c(x)$ and $\mathcal{F} \subseteq \mathcal{G}$, then $\mathcal{G} \in c(x)$;

(3) if $\mathcal{F}, \mathcal{G} \in c(x)$, then $\mathcal{F} \cap \mathcal{G} \in c(x)$.

Here $\dot{x}$ stands for the ultrafilter on $X$ generated by $\{x\}$ and the pair $(X, c)$ is called a convergence space. If $\mathcal{F} \in c(x)$, we say that $\mathcal{F}$ converges to $x$.

If $f: X \rightarrow Y$ is a map and $\mathcal{F} \in F(X)$ then $f(\mathcal{F})$ is a filter base. In general, $f(\mathcal{F})$ is not a filter but the filter generated by $f(\mathcal{F})$ is also denoted by $f(\mathcal{F})$.

Definition 2.2. Let $(X, c)$ and $\left(Y, c^{\prime}\right)$ be convergence spaces and $f: X \rightarrow Y$ a function. Then $f$ is said to be continuous at $x \in X$ if for any $\mathcal{F} \in c(x), f(\mathcal{F}) \in c^{\prime}(f(x))$. And $f$ is said to be continuous if $f$ is continuous at each point $x \in X$.

Proposition 2.3. Let $X$ be a set and $\left\{\left(X_{i}, p_{i}\right)\right\}_{i \in I}$ be a family of convergence spaces. For a family of functions $\left\{f_{i}: X_{i} \rightarrow X\right\}_{i \in I}$, define a filter $\mathcal{F}$ on $X$ converges to $x$ in $X$ if and only if for each $i \in I, f_{i}(\mathcal{F})$ converges to $f_{i}(x)$ in $X_{i}$. Then $X$ is a convergence space.

The convergence structure on $X$ is called the initial structure with respect to $\left\{f_{i}: X \rightarrow X_{i}\right\}_{i \in I}$.

Proposition 2.4. Let $X$ be a set and $\left\{\left(X_{i}, p_{i}\right)\right\}_{i \in I}$ be a family of convergence spaces. For a family of functions $\left\{f_{i}: X_{i} \rightarrow X\right\}_{i \in I}$, define a filter $\mathcal{F}$ on $X$ converges to $x$ in $X$ if and only if either $\mathcal{F}=\dot{x}$ or there are $i_{1}, \cdots, i_{n} \in I$ and a filter $\mathcal{G}_{i_{k}}$ on $X_{i_{k}}$ which converges to $y$ such that $f_{i_{k}}(y)=x$ and $\bigcap_{k=1}^{n} f_{i_{k}}\left(\mathcal{G}_{i_{k}}\right) \subseteq \mathcal{F}$. Then $X$ is a convergence space.

The convergence structure on $X$ is called the final structure with respect to $\left\{f_{i}: X_{i} \rightarrow X\right\}_{i \in I}$.

Proposition 2.5. Let $\left\{\left(X_{i}, p_{i}\right)\right\}_{i \in I}$ be a family of convergence spaces and $\prod X_{i}$ be the product endowed with the convergence structure in Proposition 2.3. with respect to $\left\{\pi_{i}: \prod X_{i} \rightarrow X_{i}\right\}_{i \in I}$. Then $\prod X_{i}$ is the product of $\left\{\left(X_{i}, p_{i}\right)\right\}_{i \in I}$. 


\section{Regular convergence spaces}

We introduce a notion of Hausdorff convergence spaces and regular convergence spaces and investigate some properties of these spaces.

Definition 3.1. [1] Let $A$ be a subset of a convergence space $X$. A point $p$ is a point of adherence of $A$ if there is a filter $\mathcal{F}$ converging to $p$ such that $F \cap A \neq \emptyset$ for all $F \in \mathcal{F}$. The set of all adherence points of $A$ is denoted by $\bar{A}$. We say that $A$ is closed if $A=\bar{A}$.

The following is easily proved.

Proposition 3.2. Let $f:(X, c) \rightarrow\left(Y, c^{\prime}\right)$ be continuous and $A \subseteq X$. Then $f(\bar{A}) \subseteq \overline{f(A)}$.

As in the topological space, a subset of a convergence space is said to be open if $A^{c}$ is closed. Note that $U$ is open if and only if $U$ belongs to every filter which converges to a point of $U[1]$.

Let $(X, \mathcal{T})$ be a topological space. Define $c_{\mathcal{T}}: X \rightarrow P(F(X))$ by $c_{\mathcal{T}}(x)=\left\{\mathcal{F} \in F(X) \mid \mathcal{N}_{x} \subseteq \mathcal{F}\right.$, where $\mathcal{N}_{x}$ is the neighborhood filter at $x$. Then $\left(X, c_{\mathcal{T}}\right)$ is a convergence space. Moreover, if $f:(X, \mathcal{T}) \rightarrow\left(Y, \mathcal{T}^{\prime}\right)$ is continuous, then $f:\left(X, c_{\mathcal{T}}\right) \rightarrow\left(Y, c_{\mathcal{T}^{\prime}}\right)$ is continuous.

Conversely, for a convergence space $(X, c)$, define $\mathcal{T}_{c}=\{U \subseteq X \mid U$ belongs to every filter which converges to a point in $U\}$. Then $\left(X, \mathcal{T}_{c}\right)$ is a topological space. Moreover, if $f:(X, c) \rightarrow\left(Y, c^{\prime}\right)$ is continuous, then $f:\left(X, \mathcal{T}_{c}\right) \rightarrow\left(Y, \mathcal{T}_{c^{\prime}}\right)$ is continuous.

By these definitions, we have the following propositions.

Proposition 3.3. (1) Let $K$ be a closed subset of a convergence space $(X, c)$. Then $K$ is closed in $\left(X, \mathcal{T}_{c}\right)$.

(2) Let $K$ be a closed subset of a topological space $(X, \mathcal{T})$. Then $K$ is closed in $\left(X, c_{\mathcal{T}}\right)$.

Definition 3.4. [1] convergence space $X$ is Hausdorff if a filter on $X$ converges to at most one point.

Definition 3.5. A convergence space $X$ is said to be weak regular if for each point $x \in X$ and each filter $\mathcal{F}$ which converges to $x$, the filter $\overline{\mathcal{F}}=\{\bar{F} \mid F \in \mathcal{F}\}$ converges to $x$. A convergence space which is hausdorff and weak regular is called a regular convergence space.

Proposition 3.6. Let $K$ be a closed subset of a convergence space $X$ and $p$ is a point with $p \notin K$. Let $\mathcal{F}$ be a filter converging to $p$. If $X$ is regular, there is an open set $U$ containing $K$ such that $U \notin \mathcal{F}$. 
Proof. Note that $K^{c}$ is open and $p \in K^{c}$. Since $X$ is regular, $\overline{\mathcal{F}}$ converges to $p$ and so $K^{c} \in \overline{\mathcal{F}}$. Hence $\bar{F} \subseteq K^{c}$ for some $F \in \mathcal{F}$. Let $(\bar{F})^{c}=U$. Then $U$ is an open set containing $K$ with $U \notin \mathcal{F}$.

Proposition 3.7. Let $(X, \mathcal{T})$ be a topological space. Let $p \in X$ and and $K$ be a closed subset of $\left(X, c_{\mathcal{T}}\right)$ with $p \notin K$. Suppose that for any filter $\mathcal{F}$ converging to $p$, there is an open set $U$ containing $K$ such that $U \notin \mathcal{F}$. Then $\left(X, c_{\mathcal{T}}\right)$ is regular.

Proof. Let $\mathcal{F}$ converge to $p$. Then $\mathcal{N}_{p} \subseteq \mathcal{F}$. Let $U$ be an open set in $\mathcal{N}_{p}$, then $p \notin U^{c}$. By assumption, there is an open set $V$ containing $U^{c}$ such that $V \notin \mathcal{F}$. Note that $V^{c} \subseteq \bar{U} \in \overline{\mathcal{F}}$. Hence $V \notin \overline{\mathcal{F}}$ and therefore $\bar{F}$ does not converge to $p$.

Proposition 3.8. Let $(X, \mathcal{T})$ be a topological space. If $\left(X, c_{\mathcal{T}}\right)$ is regular, then $(X, \mathcal{T})$ is regular.

Proof. Suppose $(X, \mathcal{T})$ is not regular. Then there is a point $p$ and closed set $K$ with $p \notin K$ such that for any open sets $U$ and $V$ with $p \in U, K \subseteq V, U \cap V \neq \emptyset$. Let $\mathcal{F}$ be a filter generated by $\{U \cap V \mid U$ : open set with $p \in U, V$ : open set with $K \subseteq V$ \}. The $\mathcal{F}$ converges to $p$ and contains all open sets containing $K$. This means that $\left(X, c_{\mathcal{T}}\right)$ is not regular by above proposition. Hence $(X, \mathcal{T})$ is regular.

Proposition 3.9. Let $X$ and $Y$ be convergence spaces and $f: X \rightarrow$ $Y$ be an initial map.

(1) If $Y$ is Hausdorff and $f$ is $1-1$, then $X$ is Hausdorff.

(2) If $Y$ is regular, then $X$ is regular.

(3) If $X$ is weak regular and $f$ is a surjection, then $Y$ is weak regular.

Proof. (1) Let $\mathcal{F}$ converges to $a$ and $b$ in $X$. Since $f$ is an initial map, $f(\mathcal{F})$ converges to $f(a)$ and $f(b)$ in $Y$. Since $Y$ is Hausdorff and $f$ is $1-1, a=b$.

(2) By (1), $X$ is Hausdorff. Let $\mathcal{F}$ converge to $x$ in $X$. Since $f$ is an initial map, $f(\mathcal{F})$ converges to $y=f(x)$. Since $Y$ is regular, $\{\overline{f(F)} \mid F \in \mathcal{F}\}$ converges to $y$. Since $F \subseteq f^{-1}(f(F)), \bar{F} \subseteq \overline{f^{-1}(f(F))}$. Hence

$$
f(\bar{F}) \subseteq f\left(\overline{f^{-1}(f(F))}\right) \subseteq \overline{f\left(f^{-1}(f(F))\right)} \subseteq \overline{f(F)}
$$

Hence $\{\overline{f(F)} \mid F \in \mathcal{F}\} \subseteq\{f(\bar{F}) \mid F \in \mathcal{F}\}$ and so $\{f(\bar{F}) \mid F \in \mathcal{F}\}$ converges to $y$. Since $f$ is an initial map, $\{\bar{F} \mid F \in \mathcal{F}\}$ converges to $x$ and hence $X$ is regular. 
(3) Let $\mathcal{F}$ converge to $y$ in $Y$. Since $f$ is initial and a surjection, $f^{-1}(\mathcal{F})$ converges to some point $x \in f^{-1}(y)$. Let $p \in f^{-1}(\bar{F})$, then $f(p) \in \bar{F}$. So there exists an ultrafilter $\mathcal{U}$ containing $F$ which converges to $f(p)$. Let $\mathcal{V}$ be an ultrafilter containing $f^{-1}(\mathcal{U})$. Then $\mathcal{V}$ converges to $p$ in $X$ and contains $f^{-1}(F)$. Thus $p \in \overline{f^{-1}(F)}$, and hence $f^{-1}(\bar{F}) \subset$ $\overline{f^{-1}(F)}$. Since $X$ is weak regular, $\left\{\overline{f^{-1}(F)} \mid F \in \mathcal{F}\right\}$ converges to $x$. Moreover, since $f$ is continuous, $\left\{f\left(\overline{f^{-1}(F)}\right) \mid F \in \mathcal{F}\right\}$ converges to $y$. Note that

$$
\bar{F}=f\left(f^{-1}(\bar{F})\right) \subseteq f\left(\overline{f^{-1}(F)}\right),
$$

and hence

$$
\left\{f\left(\overline{f^{-1}(F)}\right) \mid F \in \mathcal{F}\right\} \subseteq\{\bar{F} \mid F \in \mathcal{F}\} .
$$

Therefore $\{\bar{F} \mid F \in \mathcal{F}\}$ converges to $y$. In all, $Y$ is weak regular.

Corollary 3.10. A subspace of a Hausdorff convergence space is Hausdorff and a subspace of a regular convergence spaces is regular.

Proposition 3.11. Let $\left\{X_{i} \mid i \in I\right\}$ be a family of convergence spaces.

(1) If $X_{i}$ is Hausdorff, then $\prod X_{i}$ is Hausdorff.

(2) If $X_{i}$ is regular, then $\prod X_{i}$ is regular.

Proof. (1) Let $\mathcal{F}$ converge to $a$ and $b$ in $\prod X_{i}$. By Proposition 2.5, $\pi_{i}(\mathcal{F})$ converges to $\pi_{i}(a)$ and $\pi_{i}(b)$ for each $i \in I$. Since each $X_{i}$ is Hausdorff, $\pi_{i}(a)=\pi_{i}(b)$ for each $i \in I$. Hence $a=b$.

(2) By (1), $\prod X_{i}$ is Hausdorff. Let $\mathcal{F}$ converge to $x$ in $\prod X_{i}$. Then $\pi_{i}(\mathcal{F})$ converges to $\pi_{i}(x)$ for each $i \in I$. Since each $X_{i}$ is regular, $\left\{\overline{\pi_{i}(F)} \mid F \in \mathcal{F}\right\}$ converges to $\pi_{i}(x)$ in $X_{i}$.

Since $F \subseteq \prod \pi_{i}(F), \bar{F} \subseteq \overline{\prod \pi_{i}(F)}=\prod \overline{\pi_{i}(F)}$ and hence

$$
\left\{\prod \overline{\pi_{i}(F)} \mid F \in \mathcal{F}\right\} \subseteq\{\bar{F} \mid F \in \mathcal{F}\} .
$$

Thus $\{\bar{F} \mid F \in \mathcal{F}\}$ converges to $x$. Hence $\prod X_{i}$ is weak regular.

Proposition 3.12. Let $X$ and $Y$ be convergence spaces and $f: X \rightarrow$ $Y$ be a final map. If $Y$ is weak regular and $f$ is $1-1, X$ is weak regular.

Proof. Let $\mathcal{F}$ converge to $x$ in $X$. Then $f(\mathcal{F})$ converges to $f(x)$ in $Y$. Since $Y$ is weak regular, $\{\overline{f(F)} \mid F \in \mathcal{F}\}$ converges to $f(x)$ in $Y$. Since $f$ is final and $1-1$, there exists a filter $\mathcal{G}$ on $X$ converging to $x$ such that $f(\mathcal{G}) \subset\{\overline{f(F)} \mid F \in \mathcal{F}\}$. So, for each $G \in \mathcal{G}$ there exists an $F \in \mathcal{F}$ such that $\overline{f(F)} \subseteq f(G)$. Thus

$$
\bar{F}=f^{-1}(f(\bar{F})) \subseteq f^{-1}(\overline{f(F)}) \subseteq f^{-1}(f(G))=G .
$$


Hence $\mathcal{G} \subseteq\{\bar{F} \mid F \in \mathcal{F}\}$. Since $\mathcal{G}$ converges to $x,\{\bar{F} \mid F \in \mathcal{F}\}$ converges to $x$ in $X$. Therefore $X$ is weak regular.

\section{Function spaces}

In this section, I introduce the continuous convergence structure on $C(X, Y)$ and obtain a condition for the fibrewise regularity of the function space $C(X, Y)$.

Let $X$ and $Y$ be convergence spaces and $C(X, Y)$ be the set of all continuous functions from $X$ to $Y$. Define a filter $\mathcal{F}$ converges to $f$ in $C(X, Y)$ if and only if for any filter $\mathcal{A}$ in $X$ which converges to $x$, $\mathcal{F}(\mathcal{A})$ converges to $f(x)$ in $Y$. Then it is well known that $C(X, Y)$ with this structure is a convergence space and this structure is called the continuous convergence structure on $C(X, Y)[1]$.

Proposition 4.1. Let $X$ and $Y$ be convergence spaces. If $Y$ is Hausdorff, then $C(X, Y)$ is Hausdorff.

Proof. Let $\mathcal{F}$ converge to $f$ and $g$ in $C(X, Y)$ and $\mathcal{A}$ converge to $x$ in $X$. Then $\mathcal{F}(\mathcal{A})$ converges to $f(x)$ and $g(x)$ in $Y$. Since $Y$ is Hausdorff, $f(x)=g(x)$ and so $f=g$ in $C(X, Y)$. Hence $C(X, Y)$ is Hausdorff.

It is well known that the category consisting of convergence spaces and continuous maps between them is catesian closed [4]. Then for any convergence space $Z$ and a function $f: Z \rightarrow C(X, Y), f$ is continuous if and only if $e v \circ\left(1_{X} \times f\right): X \times Z \rightarrow Y$ is continuous, where $e v: X \times$ $C(X, Y) \rightarrow Y$ is an evaluation map which is defined by $e v(x, f)=f(x)$.

Proposition 4.2. Let $X$ and $Y$ be convergence spaces and let $K=$ $\{f \in C(X, Y) \mid f:$ constant map $\}$. Then $K$ is homeomorphic to $Y$.

Proof. Define $\phi: Y \rightarrow C(X, Y)$ by, for $y \in Y, \phi(y)=c_{y}$, where $c_{y}$ is the constant map from $X$ to $Y$ with value $y$. Clearly, $\phi$ is well-defined and injective. Note that $\phi(Y)=K$. Let $\psi: Y \rightarrow K$ be the corestriction of $\phi$. Consider the following diagram 


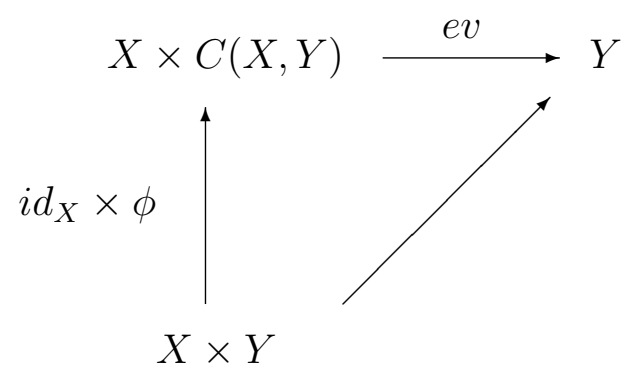

Note that $\pi_{2}=e v \circ\left(i d_{X} \times \phi\right)$, since $\pi_{2}(x, y)=y=c_{y}(x)=e v\left(x, c_{y}\right)$. Since $\pi_{2}$ is continuous, $e v \circ\left(i d_{X} \times \phi\right)$ is continuous. Hence $\phi$ is continuous, by the cartesian closedness of the category of convergence spaces. Therefore, $\psi: Y \rightarrow K$ is continuous. Now, pick an $x$ in $X$. Then we know that

$$
\psi^{-1}: K \stackrel{j}{\longrightarrow} X \times Y \stackrel{e v}{\longrightarrow} Y
$$

where $j(f)=(x, f)$ for $f \in K$. Since $j$ and $e v$ are continuous, $\psi^{-1}$ is continuous. In all, $K$ is homeomorphic to $Y$.

Proposition 4.3. Let $X$ and $Y$ be convergence spaces. If $C(X, Y)$ is Hausdorff, then $Y$ is Hausdorff.

Proof. The proof is easy by Corollary 3.10 and Proposition 4.2.

Proposition 4.4. Let $X$ and $Y$ be convergence spaces. If $Y$ is regular, then $C(X, Y)$ is regular.

Proof. By Proposition 4.1, $C(X, Y)$ is Hausdorff. Suppose $\mathcal{F}$ converges to $f$ in $C(X, Y)$. Then we have to show that $\mathcal{G}=\{\bar{F} \mid F \in \mathcal{F}\}$ converges to $f$ in $C(X, Y)$. Let $\mathcal{A}$ converge to $x$ in $X$, then it is enough to show that $\mathcal{G}(\mathcal{A})$ converges to $f(x)$ in $Y$. Since $\mathcal{F}$ converges to $f$ in $C(X, Y), \mathcal{F}(\mathcal{A})$ converges to $f(x)$ in $Y$. Hence $f(x) \in \overline{F(A)}$ for all $F \in \mathcal{F}$ and $A \in \mathcal{A}$. Since $Y$ is regular, $\{\overline{F(A)} \mid F \in \mathcal{F}, A \in \mathcal{A}\}$ converges to $f(x)$. Note that $\bar{F}(A) \subseteq \bar{F}(\bar{A}) \subseteq \overline{F(A)}$. In fact, if $y \in \bar{F}(\bar{A})$, then $y=f(x)$ for some $f \in \bar{F}$ and $x \in \bar{A}$. Then there is a filter $\mathcal{U}$ in $C(X, Y)$ converging to $f$ such that $U \cap F \neq \emptyset$ for all $U \in \mathcal{U}$ and a filter $\mathcal{V}$ in $X$ converging to $x$ such that $V \cap A \neq \emptyset$ for all $V \in \mathcal{V}$. Hence the filter $\mathcal{U}(\mathcal{V})$ is a filter in $Y$ converging to $y$ such that $U(V) \cap F(A) \cap F(A) \neq \emptyset$ 
for all $U \in \mathcal{U}$ and $V \in \mathcal{V}$ and so $y \in \overline{F(A)}$. Therefore,

$$
\{\overline{F(A)} \mid F \in \mathcal{F}, A \in \mathcal{A}\} \subseteq\{\bar{F}(A) \mid F \in \mathcal{F}, A \in \mathcal{A}\}
$$

Hence the filter $\{\bar{F}(A) \mid F \in \mathcal{F}, A \in \mathcal{A}\}$ converges to $y$ and this means that $\{\bar{F} \mid F \in \mathcal{F}\}$ converges to $f$ in $C(X, Y)$. Hence $C(X, Y)$ is regular.

By corollary 3.10 and proposition 4.2 , we have the following proposition.

Proposition 4.5. Let $X$ and $Y$ be convergence spaces. If $C(X, Y)$ is regular, then $Y$ is regular.

\section{References}

[1] E. Binz, Continuous Convergence on $C(X)$, Lecture notes in Mathematics, 469, Springer-Verlag, Berlin (1975)

[2] N. Bourbaki, General Topology, Addison-Wesley Publishing Company, (1966)

[3] H. Herrlich, Cartesian closed topological categories, Math. Coll. Univ. Cape Town. 9 (1974), 1-16.

[4] C. Y. Kim, S. S. Hong, Y. H. Hong and P. U. Park, Algebras in Catesian Closed Topological Categories, Yonsei Univ. Lecture Notes, Seoul, (1978)

[5] K. C. Min and S. J. Lee, Fibrewise convergence and exponential laws, Tsukuba J. Math. 16, No.1 (1992), 53-62.

Department of Mathematics Education

Jeju National University

Cheju 690-756, Korea

E-mail: jinwon@jejunu.ac.kr 